Originales

\title{
Intervención con un Programa de Ejercicio Físico en la empresa
}

\author{
Intervention through a Physical Exercise Program at the Company
}

\author{
M. ${ }^{a}$ Lourdes Pérez Marín', Fernando Yélamos Rodríguez ${ }^{2}$, Manuel Antonio Rodríguez Pérez ${ }^{3}$ \\ 1. CPRL de la Junta de Andalucía. C. H. Torrecárdenas. Almería. España. \\ 2. Servicio de Medicina Interna del C. H. Torrecárdenas. Almería. España. \\ 3. Departamento de Educación. Universidad de Almería. España.
}

Recibido: 29-04-15

Aceptado: 29-10-15

\section{Correspondencia}

M. ${ }^{a}$ Lourdes Pérez Marín

CPRL de la Junta de Andalucía, Almería

C) Tirso de Molina, 2

04005 Almería. España

marial.pm@live.com

Resumen

Fundamentos: Existen evidencias sobre los beneficios que conlleva la participación en Programas de Ejercicio Físico (PEF) en la empresa y por ello es importante que la participación en un PEF sea la mayor posible. El objetivo de este trabajo es hacer un estudio piloto que pueda dar información sobre el perfil de los trabajadores que participan y de los que no participan en un PEF en la empresa.

Métodos: Se han utilizado los cuestionarios validados SF-36 de calidad de vida; PSS de estrés percibido; cuestionario de autoestima más una serie de datos demográficos seleccionados. Han sido contestados por los trabajadores de una empresa que realizaba un PEF en el que participaban 17 trabajadores de 42 que forman la plantilla. El tamaño muestral es $n=31$. La participación ha sido voluntaria. La fuente de información son los trabajadores de esta empresa. Se ha realizado un estudio descriptivo transversal.

Resultados: en el grupo de trabajadores que no participaban en el PEF se encontró que la mayoría tienen un contrato de duración limitada (resultado que obtuvo significación estadística), menor nivel de estrés en el cuestionario PSS, mayor nivel de autoestima en el cuestionario de autoestima y/o en el rol físico del cuestionario SF-36; tienen una mejor percepción de salud respecto del otro grupo.

Conclusiones: a través de estudios posteriores se podría investigar la relación entre estos perfiles y la participación en PEF de entrenamiento físico en la empresa, para que la implantación de un PEF sea exitosa.

Med Segur Trab (Internet) 2015; 61 (240) 342-353

Palabras clave: Salud laboral, trabajadores, encuestas de salud, SF-36, vigilancia de la salud, actividad física, PSS, autoestima, estrés, contrato.

Abstract

Objectives: There is evidence of the benefits gained by participating in the Physical Exercise Program (PEP) in the company. The objective of this investigation is to describe the profile of employees who take part and that of employees who do not take part in the PEP. 
Methods: We used validated questionnaires, the Short Form-36 Health Survey (SF-36); PSS (stress scale perceived); self-esteem questionnaire and a series of selected demographic data. They were answered by the workers of a company who were on a PEP; 17 out of 42 who formed the workforce were participating on it. The sample size is $n=31$. Participation was voluntary. The source of information is the employees of the company. We performed a cross sectional study.

Results: we found out that most of the workers not involved in the PEP have a fixed-term contract whose result brought statistical significance in showing less stress level in the PSS questionnaire, higher selfesteem in the questionnaire of self-esteem and/or the physical role of the SF-36; they have a better perception of health compared to the other group.

Conclusions: through further studies could investigate the relationship between these profiles and the participation or non- participation in a PEP in the work place, to develop effective strategies to encourage workers to participate in PEP in the work place.

Med Segur Trab (Internet) 2015; 61 (240) 342-353

Key words: Occupational health, workers, health surveys, SF-36, bealth surveillance, physical activity, PSS, self-esteem, stress, contract. 


\section{INTRODUCCIÓN}

Se define actividad física como cualquier movimiento corporal producido por los músculos esqueléticos que exija gasto de energía mientras que el ejercicio físico es una variedad de actividad física planificada, estructurada, repetitiva y realizada con un objetivo relacionado con la mejora o el mantenimiento de uno o más componentes de la aptitud física; esta última abarca tanto el ejercicio como otras actividades que entrañan movimiento corporal y que se realizan como parte de los momentos de juego, del trabajo, de formas de transporte activas, de las tareas domésticas y de actividades recreativas ${ }^{1}$.

La inactividad física constituye el cuarto factor de riesgo más importante de mortalidad en todo el mundo ( $6 \%$ de defunciones a nivel mundial). Sólo la superan la hipertensión (13\%), el consumo de tabaco (9\%) y el exceso de glucosa en la sangre (6\%). El sobrepeso y la obesidad representan un $5 \%$ de la mortalidad mundial.

La inactividad física está cada vez más extendida en muchos países, y ello repercute considerablemente en la salud general de la población mundial, en la prevalencia de enfermedades no transmisibles (ENT) (enfermedades cardiovasculares, diabetes o cáncer) y en sus factores de riesgo, (hipertensión arterial, el exceso de glucosa en la sangre o el sobrepeso). Se estima que la inactividad física es la causa principal de, aproximadamente, 21-25\% de los cánceres de mama y de colon, $27 \%$ de la diabetes, y aproximadamente un $30 \%$ de las cardiopatías isquémicas. Además, las ENT representan actualmente casi la mitad de la carga mundial total de morbilidad. Se ha estimado que, de cada 10 defunciones, seis son atribuibles a ENT.

Está demostrado que la actividad física practicada con regularidad reduce el riesgo de cardiopatía coronaria (CPC), enfermedad cardiovascular (ECV), mejora la salud metabólica (reduciendo el riesgo de desarrollar diabetes tipo 2 y síndrome metabólico) con una relación dosis-respuesta. También reduce el riesgo de desarrollar accidentes cerebrovasculares, hipertensión arterial, cáncer de colon y cáncer de mama ${ }^{2}$. Además, la actividad física es un factor determinante en el consumo de energía, por lo que es fundamental para conseguir el equilibrio energético y el control del peso. Respecto a los trastornos mentales (depresión clínica) y salud mental reduce la ansiedad, mejora el estado de ánimo y el bienestar, aumentando la sensación de eficacia en el manejo de situaciones cotidianas ${ }^{3}$. En este sentido, existen recomendaciones a nivel mundial para la promoción de la actividad física en los diferentes países, una de las más recientes ha sido elaborada por la OMS en $2010^{2}$.

La propia Constitución Española en su redacción de 1978 relaciona la salud con la práctica de actividad física pues en su artículo 43 recoge el derecho a la salud al mismo tiempo que refleja que los poderes públicos fomentarán la educación sanitaria, la educación física y el deporte ${ }^{4}$.

Según el Informe de 2013 del Observatorio de Enfermedades Profesionales (CEPROSS) y de Enfermedades causadas o agravadas por el trabajo (PANOTRATSS) el número de bajas causadas o agravadas por enfermedades que engloban afecciones cuya evolución, mejoría o empeoramiento se relaciona con el ejercicio físico fueron: para enfermedades del aparato locomotor (3.351 casos), trastornos localizados de los nervios (345 casos), trastornos del metabolismo (148 casos), enfermedad cardiaca isquémica (93 casos), desórdenes mentales (97 casos) y enfermedades crónicas de las vías respiratorias ( 8 casos), de un total de 5.317 enfermedades causadas por el trabajo. En la categoría de enfermedad o defecto agravado por el trabajo se recogieron: enfermedades del aparato locomotor (1.147 casos), trastornos localizados de los nervios (80 casos), enfermedades de la circulación cerebral (19 casos), desórdenes mentales (13 casos), trastorno del metabolismo (5 casos) y enfermedades crónicas de las vías respiratorias (2 casos) de un total de 1.543 enfermedades. 
Por ramas de actividad, en cifras absolutas, según sexos tenemos los siguientes resultados: en hombres el mayor número de enfermedades con baja laboral se da en el sector de la "Fabricación de vehículos de motor, remolques y semirremolques", "Industria de la alimentación" en segundo lugar, "Fabricación de productos metálicos, excepto maquinaria y equipo" en tercer lugar y de la "Construcción especializada" en cuarto lugar; siendo las enfermedades profesionales (EP) más frecuentes en estas profesiones la epicondilitis y epitrocleitis; ambas pertenecientes al grupo 2 del cuadro de Enfermedades Profesionales vigente (apartado que recoge las enfermedades producidas por agentes físicos entre las que se encuentran la mayoría de las relacionadas con alteraciones del aparato locomotor).

Para las mujeres, las actividades donde se recogieron mayor número de EP fue en las "Actividades sanitarias", seguido de el "Comercio al por menor, excepto de vehículos de motor y motocicletas", los "Servicios a edificios y actividades de jardinería" en tercer lugar y la "Industria de la alimentación" en cuarto lugar, con la diferencia de que las EP más frecuentes están encabezadas por las enfermedades infecciosas (pertenecientes al grupo 3 del vigente cuadro de EP, "Enfermedades producidas por agentes biológicos") Para las "Actividades sanitarias" y para las actividades que se recogen en segundo, tercer y cuarto lugar se recogen en mayor frecuencia EP pertenecientes al grupo 2 del cuadro de EP: lesiones nerviosas por compresión, epicondilitis de codo, epitrocleitis de codo, tendinitis y tenosinovitis de muñeca y mano5.

Según la OMS, un entorno de trabajo saludable es aquel en el que trabajadores y jefes colaboran en un proceso de mejora continua para promover y proteger la salud, seguridad y bienestar de los trabajadores y la sustentabilidad del ambiente de trabajo.

Casi desde los inicios, la OMS y la Organización Internacional del Trabajo (OIT) unieron esfuerzos para avanzar en esta línea pero cómo han respondido las empresas a estos llamamientos ha sido muy diferente. Hay países que están a la cabeza en la tradición de implantar políticas de promoción de entornos saludables, en las que se incluye la implantación de un Programa de Entrenamiento Físico (PEF) para sus trabajadores, mientras que en las empresas españolas el desarrollo de estrategias como esta es menor y más reciente. Desde hace algunos años ha ido creciendo el interés por esta cuestión, principalmente desde el área de Prevención de Riesgos Laborales ${ }^{7}$.

Según el artículo 31 de la LPRL/95 los Servicios de Prevención deberán realizar una vigilancia de la salud de los trabajadores en relación con los riesgos derivados del trabajo ${ }^{6}$. Si bien a colación de esta referencia, podemos decir que el sedentarismo es un riesgo de muchos puestos de trabajo, así como los movimientos repetitivos, posturas forzadas, etc.; sin embargo, a pesar de que no hay una cita expresa en dicha ley en la que se recoja que se debe promover el ejercicio físico, existen estudios e informes que reflejan su importancia y necesidad $^{2}$.

Existe un estudio reciente y realizado en nuestro país, elaborado por el Centro Internacional de Investigación de Organizaciones de la Universidad de Navarra, en 2013, en el que se recogen los resultados del seguimiento de programas sobre promoción de la salud implantados en empresas. Dichos programas, entre otras cosas, han promovido la actividad física entre los trabajadores, la mayoría de las ocasiones con un programa de entrenamiento físico (PEF). En este estudio se recogen los resultados de empresas españolas y estadounidenses; estas últimas revelan resultados esclarecedores pues llevan implantando programas como este durante mucho más tiempo (desde los años 70 según se recoge en el estudio). Entre las conclusiones se refleja que es evidente el absentismo que producen las enfermedades cardiovasculares y cómo la falta de ejercicio perjudica a la salud global de los trabajadores. El impacto de los programas de prevención en este sentido son rentables a medio y largo plazo, por todo esto y por otras razones se concluye que la intervención en materia de salud relacionada con el ejercicio físico debe entender a la actividad física y el deporte como una herramienta y no como un fin en sí mismos y fomentar la realización de actividades deportivas con moderación y responsabilidad. Cabe destacar que iniciativas en este sentido se han implantado a raíz de estudios sobre 
patologías prevalentes en la empresa, como ocurrió en la sede de Guadalajara del grupo Mahou-San Miguel en la que se creó una Escuela de Espalda en 2001 debido a la elevada tasa de lesiones y enfermedades musculoesqueléticas prevalentes entre sus trabajadores con excelentes resultados en cuando a la disminución del número de bajas.

Entre los resultados observados a largo plazo en las empresas norteamericanas, se ha constatado la reducción del absentismo y ahorro de millones de dólares en gastos médicos en los trabajadores que han participado en este tipo de programas.

Lo que realmente hacen las empresas saludables es promover el cambio de actitud de los empleados, motivándoles a mejorar su estado de salud y creando el entorno adecuado para que puedan conseguirlo. Las empresas españolas muestran todavía, en su mayoría, una aproximación parcial a la cuestión y sitúan los temas de salud en el área de la Prevención de Riesgos Laborales. Son estas áreas las que más han avanzado, tratando de ampliar los horizontes y superar los límites de la normativa vigente en materia de prevención. Todo ello hace que tengamos en nuestra mano la posibilidad y la responsabilidad de avanzar e innovar en este sentido ${ }^{7}$.

\section{OBJETIVOS}

Presentar una metodología orientada a medir determinadas variables psicosociales asociadas a los PEFs, en base a un estudio piloto en los trabajadores de la filial española de una empresa dedicada al cultivo de semillas con su sede central en De Lier.

\section{DISEÑO DEL ESTUDIO}

La población de estudio se compone de los trabajadores de la filial española, con sede en Almería, de una empresa encargada de cultivar, exportar e investigar sobre semillas que posee su sede central en De Lier, así como otras filiales por todo el mundo, la cual fue creada en Rótterdam en 1924.

Se cuenta con la participación de los trabajadores de esta empresa que representa a un sector con gran presencia en la provincia de Almería y que cuenta con perfiles laborales que se encuentran en gran número dentro del sector de la agricultura tal y como esta se entiende en el levante andaluz, para especificar más: Jaén, Granada y Almería.

La sede almeriense de la empresa consta de una plantilla de 42 trabajadores con diferentes puestos de trabajo que se agrupan en 4 categorías:

- Finca: trabajadores de invernadero y almacén. Su trabajo es $100 \%$ físico.

- Investigación: titulados superiores que realizan su jornada laboral en el laboratorio y en la finca, aproximadamente dedicando la mitad del tiempo en cada uno de esos lugares. Su trabajo se podría considerar 50\% físico y $50 \%$ sedentario.

- Administrativos: donde se agrupan recepcionista, secretaría, jefes, directores y personal de recursos humanos. Su trabajo es casi 100\% sedentario.

- Comerciales: pasan la mayor parte del tiempo realizando desplazamientos en vehículos. Su trabajo se podría considerar casi $100 \%$ sedentario.

Dicha sede almeriense tiene un contrato de investigación con la Universidad de Almería para participar en el proyecto llamado "Entrenamiento personal en el ámbito laboral". A través de este contrato, los trabajadores que lo deseen pueden participar en dicho proyecto, es por tanto una participación voluntaria. A los participantes, tras la cumplimentación del consentimiento informado, se les realizan unas medidas antropométricas y unas pruebas de condición física; a partir de estos datos se les diseña de forma personalizada un entrenamiento que pueden realizar en un gimnasio localizado 
en la empresa o en su domicilio de 2 a 3 veces por semana durante 1 año, que es la duración del contrato con la Universidad. En el proyecto los trabajadores pueden incorporarse en el momento que lo deseen. Dicho contrato se inició en enero de 2014 y finalizó en diciembre del mismo año. Durante la duración del mismo se han ido incorporando y abandonando participantes casi todos los meses, manteniéndose casi todo el tiempo un pool de participantes que rondaba los 20 trabajadores de los 42 que conforman el número total de trabajadores de esta empresa.

\section{MATERIAL}

Se han pasado los siguientes cuestionarios:

- Consentimiento informado (obligatorio para su participación).

- Cuestionario de calidad de vida SF-36. Versión Española 1.4.

- Cuestionario de estrés percibido. Versión española (2.0). Escala de Estrés Percibido- Perceived Stress Scale (PSS)- versión completa 14 ítems.

- Cuestionario de autoestima. Versión española de la escala Rossenberg de autoestima (Martín-Albo, Nuniez et al 2007).

- Cuestionario de datos demográficos: una serie de datos que se han considerado de interés y que no formaban parte de ningún cuestionario validado.

Con la novedad de que, para hacer más fácil el acceso, cumplimentación y envío de los mismos, se han utilizado las nuevas tecnologías para hacerlos llegar a los participantes del estudio. Se han creado encuestas en soporte electrónico transcribiendo fielmente las preguntas de los cuestionarios anteriormente citados y se han enviado al e-mail de los trabajadores a través de la recepción de la empresa para que pudieran contestarlo a través de esta vía desde su propio teléfono móvil y obtener así la máxima participación. Una vez terminado el cuestionario, este era enviado automáticamente a la dirección de correo desde la que se podían recoger los resultados de las encuestas en archivo excel. Para los que no tenían acceso a estas tecnologías se ha facilitado la encuesta en soporte papel. Las encuestas eran anónimas y por tanto no recogían ninguna identidad del trabajador que las rellenaba, inclusive en las que eran remitidas desde los correos electrónicos, se recibían los cuestionarios contestados pero sin ninguna referencia sobre el remitente.

\section{PROCEDIMIENTO}

El programa de "Entrenamiento personal en el ámbito laboral" llevaba implantado en la sede almeriense de la empresa desde enero de 2014 cuando, en noviembre, se han pasado los 4 cuestionarios citados a todos los trabajadores de dicha empresa, a través de las nuevas comunicaciones con encuestas enviadas al correo electrónico de los trabajadores y en soporte papel para los que no contaban con la posibilidad de contestarlos por esa vía. Los cuestionarios añadían la pregunta de si participaban o no en el PEF.

Del total de trabajadores de la empresa $(n=42)$, la participación en el PEF en el momento en que se pasaron las encuestas era de casi la mitad (17 trabajadores) y 25 empleados no realizaban el PEF.

La población de estudio es $n=31$ y se divide en dos grupos: trabajadores que no realizan en PEF $(n=23)$ y trabajadores que sí lo realizan $(n=8)$.

Todos los datos obtenidos en este trabajo se obtuvieron a través del personal de la empresa: los datos referentes a puestos de trabajo, número de trabajadores y sexos, así como la información obtenida en los cuestionarios. Dicha información se obtuvo de forma totalmente anónima y sin tener referencias personales del trabajador que rellenó dichas encuestas. 
Algunas preguntas del cuestionario "Datos demográficos”, dado el pequeño tamaño de la muestra, tuvieron que ser modificadas agrupando sus respuestas en dos únicas posibilidades, para así facilitar el análisis de los datos. Esto ocurrió con las siguientes preguntas: edad, departamento de su puesto de trabajo, estado civil, nivel de estudios, baja laboral y duración de la baja.

Una vez obtenidos los datos se nombraron las variables y se les asignó etiquetas a los distintos valores de las variables, se pasó a una fase de depuración de los mismos, para detectar cualquier inconsistencia lógica y corregir los posibles errores. Se creó un archivo de excel desde donde se exportaron a paquete estadístico SPSS-2.0 a través del cual se procesó la información. Se estableció la significación estadística en valores inferiores a 0,05 .

Para evaluar el cuestionario de salud (SF-36. Versión española 1.4) se usó un archivo de sintaxis, calculando y codificando las variables intermedias necesarias, tras lo cual se trataron con el procedimiento descrito anteriormente.

La interpretación de resultados de las escalas del estado de salud en SF-36 la podemos ver en la tabla 1 , donde explica el resultado según si se acerca a una baja o alta puntuación en dicho test ${ }^{8}$ :

Tabla 1. Escala de estado de salud para el cuestionario SF-36

\begin{tabular}{|c|c|c|c|}
\hline Funciones & N. ${ }^{\circ}$ Preguntas & Bajo & Alto \\
\hline Función física & 10 & $\begin{array}{l}\text { Mucha limitación para realizar } \\
\text { todas las actividades físicas, } \\
\text { incluyendo bañarse o vestirse, } \\
\text { debido a la salud }\end{array}$ & $\begin{array}{l}\text { Realiza todo tipo de actividades } \\
\text { físicas, incluyendo las más } \\
\text { vigorosas, sin limitaciones, debido } \\
\text { a la salud }\end{array}$ \\
\hline Rol físico & 4 & $\begin{array}{l}\text { Problemas con el trabajo u otras } \\
\text { actividades diarias como resultado } \\
\text { de la actividad física }\end{array}$ & $\begin{array}{l}\text { Ningún problema con el trabajo } \\
\text { u otras actividades diarias como } \\
\text { resultado de la salud física }\end{array}$ \\
\hline Dolor corporal & 2 & $\begin{array}{l}\text { Dolor muy severo y } \\
\text { extremadamente limitante }\end{array}$ & $\begin{array}{l}\text { Ausencia de dolor o limitación } \\
\text { debida al dolor }\end{array}$ \\
\hline Salud general & 5 & $\begin{array}{l}\text { Evalúa la salud personal } \\
\text { actual como mala y cree que } \\
\text { probablemente empeorará en el } \\
\text { futuro }\end{array}$ & $\begin{array}{l}\text { Evalúa la salud personal como } \\
\text { excelente }\end{array}$ \\
\hline Vitalidad & 4 & $\begin{array}{l}\text { Cansancio y agotamiento todo el } \\
\text { tiempo }\end{array}$ & $\begin{array}{l}\text { Lleno de entusiasmo y energía } \\
\text { todo el tiempo }\end{array}$ \\
\hline Función social & 2 & $\begin{array}{l}\text { Interferencia frecuente y extrema } \\
\text { con las actividades sociales } \\
\text { normales debido a problemas } \\
\text { físicos y emocionales }\end{array}$ & $\begin{array}{l}\text { Realiza actividades sociales } \\
\text { normales sin interferencia debido } \\
\text { a problemas físicos o emocionales }\end{array}$ \\
\hline Rol emocional & 5 & $\begin{array}{l}\text { Problemas en el trabajo u otras } \\
\text { actividades diarias como resultado } \\
\text { de problemas emocionales }\end{array}$ & $\begin{array}{l}\text { Ningún problema con el trabajo } \\
\text { u otras actividades diarias como } \\
\text { resultado de los problemas } \\
\text { emocionales }\end{array}$ \\
\hline Salud mental & 1 & $\begin{array}{l}\text { Sensación de nerviosismo y de } \\
\text { presión todo el tiempo }\end{array}$ & $\begin{array}{l}\text { Sensación de paz, felicidad y } \\
\text { calma todo el tiempo }\end{array}$ \\
\hline $\begin{array}{l}\text { Transición de salud } \\
\text { notificada }\end{array}$ & 1 & $\begin{array}{l}\text { Cree que su salud es mucho mejor } \\
\text { ahora que hace un año }\end{array}$ & $\begin{array}{l}\text { Cree que su salud es mucho peor } \\
\text { ahora que hace un año }\end{array}$ \\
\hline
\end{tabular}

El cuestionario de estrés percibido. (Versión española (2.0). Perceived Stress Scale (PSS)- versión completa 14 ítems) se interpreta de la siguiente forma: a mayor puntuación obtenida mayor nivel de estrés percibido. El máximo que se podría obtener en dicha escala es 56 puntos y 0 el mínimo. 
El cuestionario de autoestima (CA) (Versión española de la escala Rossenberg de autoestima (Martín-Albo, Nuniez et al 2007) interpreta la puntuación obtenida del siguiente modo: si esta es menor de 25 o igual a 25 la autoestima es baja, de 26 a 29 puntos indica autoestima media y de 30 a 40 puntos autoestima elevada.

Todas las variables son cualitativas excepto el resultado de las encuestas SF 36, PSS y CA que son cuantitativas.

Respecto a las técnicas estadísticas utilizadas, se comprobó la normalidad mediante Shapiro-Wilk y ninguna variable seguía la normalidad, por ello, para establecer las diferencias de las medias de las diversas variables se utilizó la U de Mann Whitney como prueba no paramétrica para analizar los resultados de las encuestas SF-36, PSS y CA.

Respecto a los datos obtenidos a través de los datos demográficos, para comparar estas variables cualitativas, se utilizó la prueba de Chi 2 o la prueba exacto de Fisher: si el porcentaje de número de frecuencias esperadas menor a 5 fue menor o igual al $20 \%$ se utilizó Chi 2; si fue mayor al 20\% y tenemos una tabla de 2x2 se utilizó Fisher. Cuando la tabla fue mayor de 2x2 se usó Chi 2.

Para comparar los datos demográficos con los resultados de los cuestionarios validados (SF-36, PSS y CA) se usó la U de Mann Withney.

\section{RESULTADOS}

De los cuestionarios pasados a los 42 trabajadores de la empresa, fueron contestados 31, (28 a través de las nuevas comunicaciones con encuestas enviadas al correo electrónico de los trabajadores y 3 en soporte papel). Del grupo que no participaba en el PEF (25 trabajadores), se recibieron 23 encuestas contestadas) y del grupo que participaba en el PEF (17 trabajadores) contestaron 8.

- Para el cuestionario de estrés percibido (PSS), los resultados que se obtuvieron fueron los siguientes (tabla 2):

Tabla 2. Resultados cuestionario de estrés percibido (PSS)

\begin{tabular}{llcccccc}
\hline $\begin{array}{l}\text { Está realizando } \\
\text { el PEF }\end{array}$ & & n & Mínimo & Máximo & Media & Desv. Típ. & $\begin{array}{c}\text { U de Mann- } \\
\text { Whitney }\end{array}$ \\
\hline No & PSS Total & 23 & 0 & 27 & 12,43 & 5,845 & \\
& N válido (según lista) & 23 & & & & & $\mathrm{p}=0,067$ \\
\multirow{2}{*}{ Sí } & PSS Total & 8 & 1 & 24 & 16,88 & 7,827 & \\
& N válido (según lista) & 8 & & & & & \\
\hline
\end{tabular}

- Para el cuestionario de autoestima (CA) se obtuvieron los siguientes resultados (tabla 3):

Tabla 3. Resultados cuestionario de autoestima (CA)

\begin{tabular}{llcccccc}
\hline $\begin{array}{c}\text { Está realizando } \\
\text { el PEF }\end{array}$ & & n & Mínimo & Máximo & Media & Desv. Típ. & $\begin{array}{c}\text { U de Mann- } \\
\text { Whitney }\end{array}$ \\
\hline No & CA Total & 23 & 24 & 30 & 27,52 & 1,410 & \\
& N válido (según lista) & 23 & & & & & $\mathrm{p}=0,202$ \\
\multirow{2}{*}{ Sí } & CA Total & 8 & 25 & 28 & 26,88 & 1,126 & \\
& N válido (según lista) & 8 & & & & & \\
\hline
\end{tabular}


- Los resultados del SF-36 revelan los siguientes datos (tabla 4):

Tabla 4. Resultados cuestionario SF-36

\begin{tabular}{lllrlrrr}
\hline & \multicolumn{3}{c}{ Sí realiza el PEF } & \multicolumn{2}{c}{ No realiza el PEF } & p \\
\cline { 2 - 8 } & $\mathbf{n}$ & Media & Desv. Típ. & $\mathbf{n}$ & Media & Desv. Típ. & $\begin{array}{c}\text { U de Mann- } \\
\text { Whitney }\end{array}$ \\
\hline Función física (0-100) & 8 & 95 & 5,976 & 23 & 96,74 & 3,876 & 0,571 \\
Rol físico (0-100) & 8 & 87,50 & 26,726 & 23 & 98,91 & 5,213 & 0,086 \\
Dolor corporal (0-100) & 8 & 76,88 & 18,154 & 23 & 84,61 & 18,123 & 0,263 \\
Salud general (0-100) & 8 & 35,38 & 18,708 & 23 & 29,35 & 14,054 & 0,298 \\
Vitalidad (0-100) & 8 & 35 & 12,536 & 23 & 30,87 & 13,788 & 0,569 \\
Función social & 8 & 51,56 & 8,011 & 23 & 50,00 & 6,528 & 0,572 \\
Rol emocional (0-100) & 8 & 79,17 & 39,591 & 23 & 95,65 & 20,851 & 0,103 \\
Salud mental (0-100) & 8 & 25 & 9,008 & 23 & 19,13 & 12,178 & 0,219 \\
\hline
\end{tabular}

- Respecto a los datos demográficos y su relación con la participación o no en un programa de ejercicio destacan los siguientes resultados (tabla 5):

Tabla 5. Resultado cruce datos demográficos y participación en el PEF

\begin{tabular}{lccccc}
\hline & \multicolumn{5}{c}{ Participación en el PEF } \\
\cline { 2 - 6 } & \multicolumn{1}{c}{ Sí } & No & p \\
\hline Casada/o o viviendo en pareja & 4 & $30,0 \%$ & 17 & $70,0 \%$ & 0,381 \\
Separada/o, soltera/o o divorciada/o & 4 & $40,0 \%$ & 6 & $60,0 \%$ & \\
\hline Ha estado de baja alguna vez & 5 & $45,5 \%$ & 6 & $54,5 \%$ & 0,104 \\
No ha estado de baja alguna vez & 3 & $15,18 \%$ & 16 & $84,2 \%$ & \\
\hline Contrato indefinido & 7 & $43,8 \%$ & 9 & $56,3 \%$ & 0,037 \\
Duración determinada o prácticas & 1 & $6,7 \%$ & 14 & $93,3 \%$ & \\
\hline
\end{tabular}

Los resultados de la encuesta PSS que recoge el nivel de estrés percibido nos ofrecen unos datos que no tienen significación estadística pero para el estudio de esta muestra revela que la media de estrés percibido recogida entre los trabajadores que no participan en el PEF es menor que en los que sí participan.

Los datos obtenidos a través del cuestionario de autoestima (CA) tampoco obtienen significación estadística pero la media entre los dos grupos es ligeramente diferente, recogiéndose un mayor nivel de autoestima entre los trabajadores que no participan en el PEF.

El cuestionario de salud SF-36, de todos los parámetros que valora, donde se encuentran más diferencias entre los trabajadores de ambos grupos es en 5 aspectos:

- En el Rol Emocional es donde se encuentra la mayor diferencia entre las medias de ambos grupos, los trabajadores que no realizan el PEF refieren mejor puntuación al preguntarles por la presencia de problemas con el trabajo u otras actividades diarias como resultado de los problemas emocionales y esto refleja mejor situación en este aspecto respecto grupo de trabajadores que sí estaba realizando el PEF.

- En el Rol Físico, donde se valora los problemas de estos trabajadores con el trabajo u otras actividades diarias como resultado de la actividad física, obtienen una mejor percepción de salud los trabajadores que no participan en el PEF. 
- Respecto al Dolor Corporal, se refleja una menor percepción de dolor intenso y limitante entre los trabajadores de esta empresa que no realizan el PEF.

- En el aspecto de Salud Mental, se encuentra una menor puntuación obtenida en los trabajadores que no realizan el PEF, (peor estado).

- En las preguntas sobre Salud General tiene una menor puntuación el grupo de trabajadores que no realizaban en ese momento el PEF, (peor estado).

Ninguna de las diferencias entre las medias de estos parámetros tiene significación estadística, pero la que más se acerca a tener indicios de ello es la referente al Rol Físico.

Del cruce de datos demográficos con los grupos de trabajadores que realizan y no realizan el PEF en el momento de este estudio, los resultados más relevantes son los siguientes:

- En cuanto a la participación en el PEF y el estado civil en nuestra muestra, la mayoría de trabajadores que no están realizando el PEF están casados o viviendo en pareja.

- Al relacionar la participación en el PEF y el antecedente de haber tenido o no una baja laboral, la mayoría de los trabajadores que no estaban realizando el PEF no habían tenido nunca una baja laboral.

- Al cruzar los datos referentes a la participación o no en el PEF y el tipo de contrato del trabajador (indefinido o duración determinada/prácticas), la mayoría de los no participantes en el PEF tenían un contrato de duración determinada o prácticas y esta diferencia sí ha mostrado significación estadística.

\section{DISCUSIÓN}

Según estudios previos, la actividad física puede tener un efecto amortiguador sobre la experiencia de estrés y las molestias de salud así como mejor autoestima ${ }^{8}$ y se han detectado resultados de menor nivel de estrés y menor frecuencia e intensidad de síntomas de malestar físico en individuos físicamente activos ${ }^{3,9-11}$, a diferencia de lo encontrado en nuestro estudio. El uso del cuestionario de calidad de vida SF-36 ha sido utilizado en múltiples estudios de vigilancia de la salud de los trabajadores por ser una herramienta útil al tener una validez comprobada y por su utilidad para medir componentes fundamentales de la salud ${ }^{12-14}$.

Sin embargo, una de las debilidades que puede tener este estudio es no haber incluido en el cuestionario la pregunta de si realiza ejercicio físico fuera del ámbito laboral, pues no sabemos si los trabajadores que no participan en el PEF ya están realizando otra actividad física de forma espontánea fuera de la empresa y esto podría influir en los resultados de las encuestas utilizadas. Es algo importante a tener en cuenta para estudios posteriores.

Por otra parte, estas características encontradas en el grupo que realiza el PEF (menor nivel de autoestima, más sensación de estrés y peor percepción de salud) pueden ser las motivaciones de estos trabajadores para participar en el PEF (mejorar su estado de salud), y esto sí concuerda con estudios anteriores ${ }^{15}$.

Respecto a la validez interna de este estudio, podría verse afectada por el tamaño del grupo de trabajadores que están realizando el PEF y que han participado en este estudio; esta se afectaría si los 8 trabajadores no fueran representativos de los incluidos en el programa: por ejemplo más varones, de un solo puesto, etc. Esto es algo que podría corregirse si la participación en la contestación de los cuestionarios por parte de los trabajadores que realizan el PEF fuera mayor. El reducido tamaño muestral afecta a la validez externa y a la precisión, no a la validez interna. El tamaño total de la muestra $(n=31)$ es pequeño para poder realizar una extrapolación de los resultados, pero es algo que se podría corregir si aumentamos el valor de $n$, por ejemplo realizando el estudio con 
trabajadores de más de una empresa de características similares. Por último, al ser este un estudio transversal no se puede comprobar una hipótesis, pero sí plantearla.

Como conclusión de este estudio se ha obtenido que en esta muestra, la mayoría de los trabajadores que no suelen participar en un PEF en la empresa suelen ser los que poseen una o algunas de las siguientes características: tienen un contrato de duración limitada (resultado que posee significación estadística), no tienen antecedentes de baja laboral, viven en pareja, tienen un menor nivel de estrés, tienen mayor nivel de autoestima y/o en el rol físico tienen una mejor percepción de salud; mientras que los que participan tienen peores indicadores de salud.

Este estudio es sencillo y rápido; podría realizarse a mayor escala en diferentes empresas que estén realizando un PEF para obtener la información más relevante del perfil de los trabajadores que participan y de los que no participan. Posteriormente se podrían plantear hipótesis que ayuden a encontrar la relación que existe entre estos perfiles y la participación en el PEF que nos lleve en el futuro a una implantación exitosa de un PEF en la empresa.

\section{AGRADECIMIENTO}

Quiero expresar mi agradecimiento al profesor Antonio Jesús Casimiro Andújar, (Departamento de Educación, Universidad de Almería) sin él no habría sido posible este trabajo. Gracias por tu dedicación con amor y responsabilidad al estudio y fomento del deporte y el ejercicio físico.

\section{REFERENCIAS BIBLIOGRÁFICAS}

1. Estrategia mundial sobre régimen alimentario, actividad física y salud. Organización Mundial de la Salud, 2010 (http://www.who.int/dietphsicalactivity/pa/es/ visitado el 29/12/2014).

2. Recomendaciones mundiales sobre actividad física para la salud. Suiza. Organización Mundial de la Salud. 2010.

3. Remor E, Pérez-Llantada M. La relación entre niveles de la actividad física y la experiencia de estrés y de síntomas de malestar físico. R. Interam. Psicol., 2007;41 (3):313-322

4. Constitución Española, 1978. BOE n. 311 de 29 de diciembre de 1978.

5. Observatorio de enfermedades profesionales (CEPROSS) y de enfermedades causadas o agravadas por el trabajo (PANOTRATSS). Informe anual 2013. Madrid. Abril 2014.

6. Ley 31/1995, de 8 de noviembre, de Prevención de Riesgos Laborales. BOE n. ${ }^{\text {o }}$ 269 10/11/1995

7. IESE Bussiness School-Universidad de Navarra. Productividad y empresa saludable. Madrid. 2013

8. Cantero M, Pérez Y, Portela Y, Rodríguez E. Deporte, actividad física y salud de los trabajadores. Su importancia para la productividad en la Universidad de las Ciencias Informáticas. EmásF, 2014;(28):61-73

9. Jiménez M, Martínez P, Miró E, Sánchez A. Bienestar psicológico y hábitos saludables: ¿están asociados a la práctica de ejercicio físico? Int j Clin Health Psicol., 2007;8(1):185-202

10. Miguel JM, Schweier I, de las Mozas O, Hernández JM. Efecto del ejercicio físico en la productividad laboral y el bienestar. Revista de Psicología del Deporte, 2011;20(2):580-604

11. Escribà-Agüir V. Ambiente psicosocial y salud de los trabajadores. Arch Prev Riesgos Labor, 9(1):6-9

12. Leme J, Meyer E. Efectos de la gimnasia laboral en la calidad de vida de los trabajadores. Cienc. Trab, 2008;10(29):100-105

13. Casimiro A, Artés E, Muyor J, Rodríguez M. Incidencia de un programa de actividad física en la calidad de vida de los trabajadores en su ámbito laboral. Arch Med Deporte 2011;28(144):168-167

14. García A, Santibáñez M, Soriano G. Utilización de un cuestionario de salud percibida (SF-36) en vigilancia de la salud de los trabajadores. Arch Prev Riesgos Labor 2004;7(3):88-98

15. Capdeúla U, Niñerola J, Pintanel M. Motivación y actividad física: el autoinforme de motivos para la práctica de ejercicio físico (AMPEF). Revista de Psicología del Deporte, 2004;13(1):55-74 


\section{ANEXO 1}

\section{- Datos demográficos.}

Las siguientes preguntas tratan sobre información demográfica.

1. ¿En qué Departamento se encuentra su puesto de trabajo?

Finca

Investigación

Administrativo/a

Comercial

4. ¿De qué tipo es la duración de su contrato? *

Marca solo un óvalo.

Mi contrato es indefinido

Mi contrato es de duración

determinada

Mi contrato es de prácticas

5. Edad*

Marca solo un óvalo.

Hasta 25

De 26 a 35

De 36 a 45

De 46 a 55

Más de 55

6. Sexo *

Marca solo un óvalo.

Mujer

Hombre

7. ¿Cuál es el nivel de estudios más alto que ha completado? *

Marca solo un óvalo.

Sin estudios

Escolariad

Educación primaria: graduado

escolar o FPI

Educación secundaria: bachillerato,

FP II

Diplomatura universitaria,

Arquitectura técnica, Ingeniería

técnica, etc.

Licenciatura universitaria,

Arquitectura, Ingeniería

Otro:
8. Estado civil *

Marca solo un óvalo.

Soltero/a

Casado/a o viviendo en pareja

Separado/a o divorciado/a

Viudo/a

9. ¿Ha estado de baja alguna vez? * Marca solo un óvalo.

Sí

No

10. Si la respuesta anterior es sí: ¿cuántas veces ha estado de baja?

(respuesta libre)

11. Si ha estado de baja alguna vez, ¿de cuanta duración ha sido la baja más larga?

(respuesta libre)

12. Si está realizando el programa de entrenamiento: ¿ha estado de baja mientras lo estaba realizando?

Marca solo un óvalo.

Sí

No

13. Si en la pregunta anterior la respuesta es sí, ¿cuantas veces ha estado de baja?

Marca solo un óvalo.

En una ocasión

En más de una ocasión

14. Si ha estado de baja mientras realizaba el programa de entrenamiento, ¿cuanto tiempo ha durado? 